the worst case, it appears grossly to be like school paste of the kind provided to me in the dark ages. Bottom line: when you weigh out PTA with much water and add it to a stain formulation, the stain will perform as though no PTA has been added. I've seen the effect in Pap EA formulations, the origins of which are rooted in Mallory's trichrome stain. In such instances, the EA is awful. Light green and eosin stain the same cells so that they are muddy and totally unacceptable, instead of distinctly green and red.

Therefore, dry PTA by removing the screw cap of the bottle and heating the bottle in a warm oven overnight. Prepare a $20 \%$ (wtivol) solution of PTA in $95 \%$ alcohol. Thereafter, volumetrically, rather than gravimetrically, dispense the volume required to provide the desired weight of PTA. This approach is convenient. It avoids the hygroscopicity of PTA and facilitates stain preparation. Each $10 \mathrm{~mL}$ contains $2 \mathrm{gm}$ PTA. For my EA, for example, use $20 \mathrm{~mL}$ per $980 \mathrm{~mL}$ of solution to provide the recommended $4 \mathrm{gm}$ per $\mathrm{L}$

P.S.: If anybody knows why Mallory had the insight to use PTA, I'd love to know. Why on earth would anybody choose PTA for this purpose? Mallory must have had a reason.

suspect this same tip would apply equally to phosphomolybdic acid (PMA), which is also a hydrate of variable and uncertain composition, vis-à-vis, water content. I'm not positive, however, as I've not worked with PMA.

Quoting Lillie and Fullmer ( $p$ 699): "Although there has been considerable dispute as to the relative efficiency of phosphomolybdic and phosphotungstic acids and as to the proper concentration and exposure time, it seems to make little difference whether one uses one, the other, a combination of both, or neither as long as sections are treated with acid before and during the fiber stain."

References:

Lillie, R.D. and H.M. Fulimer. 1976. Histopathologic Technic and Practical Histochemistiy. 1st ed. McGraw-Hill, New York.

Puchtier, H. 1958 No title. J Histochem Cytochem. 6:265.

Gary Gill, Diagnostic Cytology Laboratories, Inc., Indianapolis IN

\section{Tips On Thick Mounting Media And Refractive Index Problems.}

Whole mounts of small arthropods, fungi, and other organisms with chitinous cuticles often have problems with contrast. This problem is derived from the similarity of the refractive indices of chitin and your mounting material. I did some work on this issue many years ago and offer the following solutions. These ideas also apply more generally to thick-mount specimens.

1) First, an observation: the refractive index (RI) of chitin is about the same as immersion oil (1.515). As I remember, we tried immersion oil as a mounting material and the chitin all but disappeared.

2) Concerning refractive index changes: you don't have to have much of a refractive index change to increase the contrast. Cargille has a whole slew of mounting oils from which to choose. Water $(R I=1.33)$ could also be used as a mounting medium. A more permanent mount may be made by making sure that the drop of water does not exceed the edge of the coverslip, then sealing with something like nail polish.

3) Concerning spherical aberration: the microscope's optics expect to see very specific components in the sample prep "sandwich". All the changes which cause spherical aberration (among other things) are derived from Snell's law (see any basic physics book). A higher refractive index will actually accentuate the problem, not solve it. The first thing to do to optimize imaging is to check the barrel of the objectives you are using and make sure that they say " 0.17 " for coverslip thickness (to order, a number $11 / 2$ is the equivalent). Anything thicker will contribute significantly to spherical aberration. Secondly, when using the higher RI mounting materials, try an objective with a "coverslip correction collar". Adjusting the collar may reduce the spherical aberration problem.
3) Colloids are serious scatterers. The resulting scatter will create glare and haze which obscures information.

For more specifics on coverslips, mounting media, and spherical aberration, may we suggest the book Optimizing Light Microscopy for Biological and Clinical Laboratories? Details are available at our website: http://www.MME-Microscopy. com/education

Barbara Foster, Microscopy/Microscopy Education

\section{Indicator For Water In Absolute Alcohol:}

Dri-Rite may be used in absolute alcohol to detect the presence of water. This was a suggestion of a former employee of the Texas Children's Hospital, Sybil Horak.

Adding Dri-Rite (blue colored desiccant pellets) to absolute alcohol will turn the alcohol blue. The alcohol should be filtered to remove the solid pellets before use. The absolute alcohol is put on the processor, or wherever absolute alcohol is used, and it. will turn pink when water gets in it. The blue dye will also turn all your tissues blue in the case of small tissue biopsies, the blue color makes all bits and pieces visible. (Pathologists seem to like it because they do not have to stain the gut biopsies with hematoxylin anymore.)

It is important to note that even though the tissues turn blue, the dye will not interfere with any routine, special, or immunohistochemical staining. The blue color also helps with orienting skin biopsies.

However, when we used it we experienced two drawbacks. First, our newer inexperienced histotechs tried to use the blue dye as an indicator of when they had achieved full face of the tissue. They figured once the blue dye was gone, they had full face and could start taking sections. The dye does not penetrate the whole tissue unless the specimen is small. In the case of large tissues this was true, but with small biopsies one could cut away all the tissue without the blue color going away. Perhaps a way to avoid this problem is to add sufficient absolute alcohol so that the blue coloring is a pale color rather than a dark blue.

The second problem we found was pathologists who routinely use hematoxylin or Mercurichrome to mark specific sites as landmarks: those spots were obscured by the blue color. Again perhaps lessening the intensity of the blue coloring would also take care of this.

Using Dri-Rite as a water indicator was met with mixed reviews here, but try it and see for yourself.

Cheryl Rehfeld, Texas Children's Hospital, Houston, TX

\section{A Cheap Holder for Small Reagent Bottles}

I have had problems with small quantities of reagents being accidentally tipped over; especially when working with plastics and I wanted to pipette resins from their mixing beaker to a mold. To prevent the problem, I cut an appropriate sized hole in a lid of one of the boxes that microscope slides are sent in. The tube or small beaker can then be placed in the hole and this prevents tipping-over. The holder is free and easy to replace when it gets dirty.

\section{John Tarpley, Amgen Inc, Thousand Oaks, California}

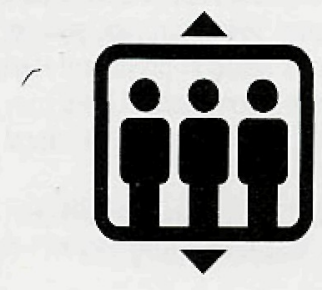

